\title{
Forced Oscillations in the System of Anisotropic Stripe-half-plane with Hard and Sliding Connection of Media. Comparative Characteristics of Properties of Energy Fields
}

\author{
Michael Remizov, Rostov-on-Don State University of Civil Engineering
}

\begin{abstract}
This is the first research which considered the problem of forced oscillations strip connected with the half-plane where both scopes were supposed to be different anisotropy to the orthorhombic crystal system. Researchers used the method of contour integration in the fields of displacements and a comparative analysis of the properties of the energy flows in hard and sliding connection of these medium.
\end{abstract}

Keywords - Anisotropic medium, contour integration, distribution of energy flow, flow of energy, Fourier transforms, half-plane, rigid and slide connection, strip.

\section{INTRODUCTION}

The influence of different pads located along the edge of the semi-infinite environment, was seen repeatedly. For anisotropic models of semi-infinite substrates the influence of the properties of border discontinuities on the dispersion curves and surface waves was investigated [1], [2]. The authors of [3] and [4] studied the acoustic effects observed in the elastic wave propagation in layered anisotropic media.

However, analysis of the energy transferred by anisotropic medium with overlay, was presented in smaller publications, among them [5] can be mentioned, where the energy characteristics of wave fields in multilayered anisotropic composites and estimation of the distribution of the portable power mod was investigated.

It is the first time that the researchers considered the problem of forced oscillations of strip connected with the halfplane, where both scopes were supposed to be of different anisotropy up to the orthorhombic crystal system inclusive of their hard and sliding connections. The defining move for each of the areas addressed issues associated with energy transfer and dependence of this characteristic length of the segment action of surface loading. The study first showed the relationship between the flows of energy transmitted in each of the medium separately, with respect to the total flow supplied to the infinite layer in the half-plane through the area dimension of loading of different lengths. A comparative analysis of properties of energy flows in hard and sliding connection is given. For numerical studies and examples, we used the following anisotropic material of strip and half-plane, respectively: titanate barium and beryllium.

\section{FORMULATION OF THE PROBLEM}

The following dynamic problem is considered. In fields $V^{(1)}=\left\{\left|x_{1}\right| \leq \infty, \quad-h \leq x_{3} \leq 0\right\}, V^{(2)}=\left\{\left|x_{1}\right|<\infty, \quad x_{3} \geq 0\right\}$ we will seek the solution of the differential equations of motion, transformed by Fourier in the assumption of the existence of the mode steady-state oscillations (time-dependent factor $e^{i \omega t}$ is omitted):

$$
\begin{aligned}
& \left(-C_{11}^{(k)} \alpha+C_{55}^{(k)} \partial_{3}^{2}+\rho^{(k)} \omega^{2}\right) U_{1}^{(k)}-i \alpha\left(C_{55}^{(k)}+C_{13}^{(k)}\right) \partial_{3} U_{3}^{(k)}=0, \\
& -i \alpha\left(C_{55}^{(k)}+C_{13}^{(k)}\right) \partial_{3} U_{1}^{(k)}+\left(-C_{55}^{(k)} \alpha^{2}+C_{33}^{(k)} \partial_{3}^{2}+\rho^{(k)} \omega^{2}\right) U_{3}^{(k)}=0,
\end{aligned}
$$

where $k=1,2$ defines the system for strip and half-plane, respectively.

Boundary conditions and the conditions that characterize the type of interaction of these media are the following:

1) The case of rigid connection:

$$
\left.\begin{array}{l}
\left.\begin{array}{l}
U_{1}^{(1)}\left(x_{1}, \alpha\right)=U_{1}^{(2)}\left(x_{1}, \alpha\right) \\
U_{3}^{(1)}\left(x_{1}, \alpha\right)=U_{3}^{(2)}\left(x_{1}, \alpha\right) \\
\sigma_{13}^{(1)}\left(x_{1}, \alpha\right)=\sigma_{13}^{(2)}\left(x_{1}, \alpha\right) \\
\sigma_{33}^{(1)}\left(x_{1}, \alpha\right)=\sigma_{33}^{(2)}\left(x_{1}, \alpha\right)
\end{array}\right\} \quad x_{3}=0 ; \\
\sigma_{13}^{(1)}\left(x_{1}, \alpha\right)=0 \\
\sigma_{33}^{(1)}\left(x_{1}, \alpha\right)=F(\alpha)
\end{array}\right\} \quad x_{3}=-h, \quad x_{1} \in[-a ; a] .
$$

2) The case of sliding connection:

$$
\begin{aligned}
& U_{3}^{(1)}\left(x_{1}, \alpha\right)=U_{3}^{(2)}\left(x_{1}, \alpha\right) \\
& \left.\sigma_{13}^{(1)}\left(x_{1}, \alpha\right)=\sigma_{13}^{(2)}\left(x_{1}, \alpha\right)=0\right\} \quad x_{3}=0 \text {; } \\
& \sigma_{33}^{(1)}\left(x_{1}, \alpha\right)=\sigma_{33}^{(2)}\left(x_{1}, \alpha\right) \\
& \left.\begin{array}{l}
\sigma_{13}^{(1)}\left(x_{1}, \alpha\right)=0 \\
\sigma_{33}^{(1)}\left(x_{1}, \alpha\right)=F(\alpha)
\end{array}\right\} \quad x_{3}=-h, \quad x_{1} \in[-a ; a] .
\end{aligned}
$$

The statement of the problem completes the selection condition of the only solution, which here is the limiting absorption principle [10]. 
After that the standard technique of solution is applied. Imagine Fourier transforms for strip and half-plane in the form

$$
\underline{U}^{(1)}\left(\alpha, x_{3}\right)=\underline{A} e^{\gamma x_{3}}, \quad \underline{U}^{(2)}\left(\alpha, x_{3}\right)=\underline{B} e^{-i \beta x_{3}},
$$

and substituting (4) with (1) where $k=1,2$, respectively. There will be two systems of homogeneous algebraic equations for the component vectors $\underline{A}, \underline{B}$. A nontrivial solution of these systems turns to a zero specifier. These conditions determine the expression of characteristic numbers $\gamma_{m}, \beta_{m}, m=1-4$.

Omitting the details of calculations, we give the expression $\gamma_{k}(\alpha), \beta_{k}(\alpha)$ to dimensionless forms. For this we introduce the dimensionless elastic constants

$$
b_{m n}^{(1)}=C_{m n}^{(1)} / C_{33}^{(1)}, \quad m, n=1, \ldots, 6
$$

Then the formula for the roots takes the form of

$$
\begin{array}{ll}
\tilde{\beta}_{1,3}(\tilde{\alpha})= \pm \sqrt{G_{1}^{(2)}(\tilde{\alpha})}, & \tilde{\beta}_{2,4}(\tilde{\alpha})= \pm \sqrt{G_{2}^{(2)}(\tilde{\alpha})}, \\
\tilde{\gamma}_{1,3}(\tilde{\alpha})= \pm i \sqrt{G_{1}^{(1)}(\tilde{\alpha})}, \quad \tilde{\gamma}_{2,4}(\tilde{\alpha})= \pm i \sqrt{G_{2}^{(1)}(\tilde{\alpha})},
\end{array}
$$

where

$$
\begin{aligned}
& G_{1}^{(k)}(\tilde{\alpha})=1+b_{55}^{(k)}-\tilde{\alpha}^{2} d^{(k)}+\sqrt{D_{k}(\tilde{\alpha})} \\
& G_{2}^{(k)}(\tilde{\alpha})=1+b_{55}^{(k)}-\tilde{\alpha}^{2} d^{(k)}-\sqrt{D_{k}(\tilde{\alpha})} \\
& \tilde{\alpha}=\alpha / \Omega, \quad \Omega=\omega \sqrt{\frac{\rho^{(k)}}{c_{33}^{(k)}}}, \quad b_{k m}^{(k)}=\frac{C_{k m}^{(2)}}{C_{33}^{(k)}}, \quad \tilde{\rho}^{(k)}=\frac{\rho^{(k)}}{c_{33}^{(k)}} ; \\
& \tilde{\beta}_{k}=\beta_{k} \sqrt{\frac{2 b_{55}^{(2)}}{\tilde{\rho}^{(2)}}}, \quad \tilde{\gamma}_{k}=\gamma_{k} \sqrt{\frac{2 b_{55}^{(1)}}{\tilde{\rho}^{(1)}}} \\
& d^{(k)}=b_{11}^{(k)}-2 b_{55}^{(k)} b_{13}^{(k)}-\left[b_{13}^{(k)}\right]^{2} .
\end{aligned}
$$

Thus the discriminant $D_{k}(\tilde{\alpha})$ is expressed by formulas

$$
\begin{aligned}
& D_{k}(\tilde{\alpha})=\left(1-b_{55}^{(k)}\right)+\tilde{\alpha}^{(4)} d_{2}^{(k)}-2 \tilde{\alpha}^{2} d_{3}^{(k)} ; \\
& d_{2}^{(k)}=\left[d^{(k)}\right]^{2}-4 b_{11}^{(k)}\left[b_{55}^{(k)}\right]^{2} ; \\
& d_{3}^{(k)}=d^{(k)}\left(1+b_{55}^{(k)}\right)-2 b_{55}^{(k)}\left(b_{11}^{(k)}+b_{55}^{(k)}\right) .
\end{aligned}
$$

For convenience, the tilde over $\gamma_{k}, \beta_{k}$ further will be omitted.

When considering characteristic functions (5) and (6) in complex plane $\zeta=\sigma+i \eta(\sigma=\tilde{\alpha})$ further it is assumed that the branches of the roots are selected according to the conditions

$$
\operatorname{Re} \sqrt{D_{k}(\zeta)}>0, \quad \operatorname{Re} \sqrt{G_{m}^{(k)}(\zeta)}>0, \quad k, m=1,2 .
$$

In contrast, the solutions for stripes, involving all four roots of the characteristic equation, the half-plane must choose two values $\beta_{m}$ that satisfy the attenuation of the waves at infinity $\left(x_{3} \rightarrow \propto\right)$

$$
\operatorname{Im} \beta_{m}<0 ; m=1,2 \text {. }
$$

It is easy to carry it out for those $\beta_{m}$, which take complex values. If $\beta_{m}$ is real, the limiting absorption principle is used, which allows to build the only solution for the half-plane. This principle, as it is known, is that instead of the parameter $\omega$, $\omega-i \varepsilon, \varepsilon>0$ is introduced and the roots $\beta_{m}$, satisfying the condition (9) are taken.

Note that under the values of $\beta_{1}, \beta_{2}$, one should understand a couple of roots involved in the solution with the required sign before their external radicals from (5) in order to meet the condition (9).

So, we get the general expression of the transformant solution for strip and half-plane, respectively:

$$
\begin{aligned}
& \underline{U}^{(1)}\left(\alpha, x_{3}\right)=\sum_{k=1}^{4} C_{k} \underline{A}^{(k)} e^{\gamma_{k} x_{3}}, \\
& \underline{U}^{(2)}\left(\alpha, x_{3}\right)=\sum_{k=1}^{4} \tilde{C}_{k} \underline{B}^{(k)} e^{-i \beta x_{3}} .
\end{aligned}
$$

Now we will use the boundary conditions to determine the unknown constants $C_{k}, \tilde{C}_{k}$, included in (10) for each type of connection. The advanced matrix of these heterogeneous systems takes the following forms:

1) The case of rigid connection:

$$
\left(\begin{array}{cccccc|c}
a_{11} & a_{12} & a_{13} & a_{14} & 0 & 0 & 0 \\
a_{21} & a_{22} & a_{23} & a_{24} & 0 & 0 & F \\
A_{1}^{(1)} & A_{1}^{(2)} & A_{1}^{(3)} & A_{1}^{(4)} & -B_{1}^{(1)} & -B_{1}^{(2)} & 0 \\
1 & 1 & 1 & 1 & -1 & -1 & 0 \\
a_{51} & a_{52} & a_{53} & a_{54} & a_{55} & a_{56} & 0 \\
a_{61} & a_{62} & a_{63} & a_{64} & a_{65} & a_{66} & 0
\end{array}\right)
$$

2) The case of sliding connection:

$$
\left(\begin{array}{cccccc|c}
a_{11} & a_{12} & a_{13} & a_{14} & 0 & 0 & 0 \\
a_{21} & a_{22} & a_{23} & a_{24} & 0 & 0 & F \\
0 & 0 & 0 & 0 & -a_{55} & -a_{56} & 0 \\
1 & 1 & 1 & 1 & -1 & -1 & 0 \\
a_{51} & a_{52} & a_{53} & a_{54} & 0 & 0 & 0 \\
a_{61} & a_{62} & a_{63} & a_{64} & a_{65} & a_{66} & 0
\end{array}\right),
$$


where

$$
\begin{aligned}
& \left\{\begin{array}{l}
a_{1 k}=\left(-i \alpha A_{3}^{(k)}+A_{1}^{(k)} \gamma_{k}\right) b_{55}^{(1)} e^{\gamma_{k} h} ; \\
a_{2 k}=\left(-i \alpha b_{13}^{(1)} A_{1}^{(k)}+A_{3}^{(k)} \gamma_{k}\right) e^{\gamma_{k} h} ; \\
a_{5 k}=\left(-i \alpha A_{3}^{(k)}+A_{1}^{(k)} \gamma_{k}\right) b_{55}^{(1)} ; \\
a_{6 k}=-i \alpha b_{13}^{(1)} A_{1}^{(k)}+A_{3}^{(k)} \gamma_{k} ; \\
a_{55}=i b_{55}^{(2)}\left[\alpha B_{3}^{(1)}+\beta_{1} B_{1}^{(1)}\right] ; \\
a_{56}=i b_{55}^{(2)}\left[\alpha B_{3}^{(2)}+\beta_{2} B_{1}^{(2)}\right]
\end{array}\right. \\
& \left\{\begin{array}{l}
a_{65}=i\left[\alpha B_{1}^{(1)} b_{13}^{(2)}+\beta_{1} B_{3}^{(1)}\right] \\
a_{66}=i\left[\alpha B_{1}^{(2)} b_{13}^{(2)}+\beta_{2} B_{3}^{(2)}\right] \\
k=\overline{1,4}, \quad \gamma_{1}=\gamma_{1}, \gamma_{2}=-\gamma_{1}, \gamma_{3}=\gamma_{2}, \gamma_{4}=-\gamma_{2} .
\end{array}\right.
\end{aligned}
$$

Solving the system (11) and (12) relative to $C_{k}, \tilde{C}_{k}$, will provide solutions for both media in the form of the following transformant:

$$
\underline{U}^{(1)}=\sum_{n=1}^{4} \frac{\tilde{D}_{n}(\alpha)}{D_{0}^{\varepsilon}(\alpha, \omega)} \underline{A}^{(n)} e^{\gamma_{n} x_{3}}, \underline{U}^{(2)}=\sum_{k=1}^{2} \frac{\hat{D}_{k}(\alpha)}{D_{0}^{\varepsilon}(\alpha, \omega)} \underline{B}^{(k)} e^{-i \beta_{k} x_{3}}
$$

Applying the inverse transform to (14) we can write the solution to the strip and half-plane $x_{3} \geq 0$ in the integral form used below.

$$
\begin{aligned}
& \underline{u}^{(1)}=\frac{1}{2 \pi} \int_{-\infty}^{\infty}\left\{\sum_{n=1}^{4} \frac{\tilde{D}_{n}(\alpha)}{D_{0}^{\varepsilon}(\alpha, \omega)} \underline{A}^{(n)} e^{\gamma_{n} x_{3}-i \alpha x_{1}}\right\} d \alpha, \\
& \underline{u}^{(2)}=\frac{1}{2 \pi} \int_{-\infty}^{\infty}\left\{\sum_{k=1}^{2} \frac{\hat{D}_{k}(\alpha)}{D_{0}^{\varepsilon}(\alpha, \omega)} \underline{B}^{(k)} e^{-i \beta_{k} x_{3}-i \alpha x_{1}}\right\} d \alpha .
\end{aligned}
$$

Further applied known approach to the calculation of improper integrals (15) and (16) is based on the method of contour integration in complex plane. The feasibility of this process stems from the fact that for the elastic field asymptotic effective evaluation can be obtained far from sources of the disturbance area, for large $R=\sqrt{x_{1}^{2}+x_{3}^{2}}$. For performing contour integration and cuts, on the banks of which the sheets of the Riemann surface are connected, where the functions $\beta_{k}(\alpha), \quad k=1,2,3,4$ are uniquely identified, the limiting absorption principle is used.

Integrand expression in (15) and (16), after the introduction according to the principle of limiting absorption of small friction $\varepsilon>0$, will have four branch points

$$
\zeta_{k}= \pm\left(\alpha_{k}-i \eta_{k}\right), \eta_{k}>0 ;\left|\alpha_{k}\right|>>\left|\eta_{k}\right|, k=1,2
$$

and a finite number of poles $\stackrel{o}{\zeta}= \pm\left(\stackrel{o}{\alpha_{n}}-i \stackrel{o}{\eta_{n}}\right)$ :

$$
D_{0}\left(\stackrel{o}{\zeta}, \omega_{0}\right), \quad n=1,2, \ldots, N
$$

Positive branch points are shifted in the lower complex half-plane, negative on the top. If we consider the shift from real axis poles, then imagine implicit function $\tilde{D}_{0}\left(\alpha_{n}, \omega_{0}\right)=0$ in the form

$$
\stackrel{o}{\alpha_{n}}=\chi\left(\omega_{0}\right) .
$$

Then, when you replace $\omega_{0} \Leftrightarrow \omega_{0}-i \varepsilon$, the new pole takes the form

$$
\begin{aligned}
& \stackrel{o}{\zeta_{n}}=\chi\left(\omega_{0}-i \varepsilon\right)=\chi\left(\omega_{0}\right)+\chi \frac{\partial \chi}{\partial \varepsilon} ; \\
& \frac{\partial \chi}{\partial \varepsilon}=\chi\left(\omega_{0}\right)+\frac{\partial \chi\left(\omega_{0}-i \varepsilon\right)}{\partial\left(\omega_{0}-i \varepsilon\right)} \cdot \frac{\partial\left(\omega_{0}-i \varepsilon\right)}{\partial \varepsilon}=\stackrel{o}{\alpha_{n}-i \frac{\partial \alpha_{n}}{\partial \omega_{0}}} \\
& \stackrel{o}{\zeta_{n}}=\stackrel{o}{\alpha_{n}}-i \frac{\partial \stackrel{o}{\alpha_{n}}}{\partial \omega_{0}} .
\end{aligned}
$$

From (18) we see that the shift of the pole with real axis is determined by the sign of the derivative of $\frac{\partial \alpha_{n}}{\partial \omega_{0}}$.

The next step is the deciding on the choice of spending cuts in the complex plane $\zeta$, allowing to select definite branch functions from (15) and (16), corresponding to the limiting absorption principle: when considering $\beta_{n} \in C$, choose roots that satisfy the attenuation of waves at infinity $x_{3} \rightarrow \infty$, so,

$$
\beta_{n}: \quad \operatorname{Im} \beta_{n}<0, \quad n=1,2 .
$$

Before proceeding to further discussion, let us pay attention to the following. The numerical results presented in this work belong to such a half-plane anisotropy, when in terms of dimensionless parameters of elasticity $\alpha, \beta, \gamma$ and the dimensionless constant $b_{m n}$, introduced for the half-plane above,

$$
\alpha=\frac{b_{55}}{b_{11}}, \beta=b_{55}, \gamma=\frac{b_{11}-2 b_{55} b_{13}-\left(b_{13}\right)^{2}}{b_{11}}=\frac{d}{b_{11}},
$$

this type is defined as the second one, according to $\mathrm{V}$. Budaev's classification in [11]:

Type II $\alpha(1+\beta)<\gamma, 2 \sqrt{\alpha \beta}$ (for example, beryllium and barium titanate).

According to the radiation conditions the only possible way of spending cuts was obtained in work [6] and [7] and used in [8] and [9].

Thus, given that in case $x_{1}<-a$ calculations are similar, forming the General contour of integration, consisting of a circle of large radius in the lower complex half-plane, a segment of real axis and two loops using the Cauchy theorem and Lemma Jordan, come to equality: 


$$
\begin{aligned}
& \int_{-\infty}^{\infty} \underline{\Phi}^{(m)}(\alpha) d \alpha=\left.i \sum_{k=1}^{N} \operatorname{Res} \underline{\Phi}^{(m)}(\zeta)\right|_{\zeta=\zeta_{k}^{0}}- \\
& -\frac{1}{2 \pi} \int_{L_{1}} \Phi^{(m)}(\zeta) d \zeta-\frac{1}{2 \pi} \int_{L_{2}} \underline{\Phi}^{(m)}(\zeta) d \zeta,
\end{aligned}
$$

where

$$
\begin{aligned}
& \underline{\Phi}^{(1)}(\alpha)=\sum_{n=1}^{4} \frac{\tilde{D}_{n} \underline{A}^{(n)}}{D_{0}^{\varepsilon}} e^{\gamma_{n} x_{3}-i \alpha x_{1}} \\
& \underline{\Phi}^{(2)}(\alpha)=\sum_{n=1}^{2} \frac{\hat{D}_{n} \underline{B}^{(n)}}{D_{0}^{\varepsilon}} e^{-i \beta_{n} x_{3}-i \alpha x_{1}}
\end{aligned}
$$

are functions to strip and half-plane, respectively, $L_{1}, L_{2}$ are paths along the banks of the sections and encircling the branch point $\alpha_{1}, \alpha_{2}: \quad \beta_{m}\left(\alpha_{m}\right)=0, \quad m=1,2$.

\section{ANALYSIS OF ENERGY FLOW}

One of the main questions of the present study was the energy of received fields in both media. The dependence of energy characteristics from the properties of driving force and connection kind of the strip and half-plane was determined. General expression of the average period power stream supplied to the infinite layer on the half-plane was considered. The average period power stream $\bar{W}$ loaded through normal forces pad $\left|x_{1}\right| \leq a, x_{3}=-h$ can be defined by the formula [12]:

$$
\bar{W}=\frac{\omega}{2} \int_{-a}^{a} g\left(x_{1}\right) \operatorname{Im}\left[u_{3}^{(1)}\left(x_{1},-h\right)\right] d x_{1}
$$

According to (22), to calculate $\bar{W}$, you need to know $\operatorname{Im}\left[u_{3}^{(1)}\left(x_{1},-h\right)\right]$ for $\left|x_{1}\right| \leq a$. To determine $u_{3}^{(1)}\left(x_{1},-h\right)$ the method of contour integration is applicable to the calculation of the integral (15) after finding out the properties of $F(\alpha)$ which Fourier transformed from the specified function of the load:

$$
F(\alpha)=\int_{-a}^{a} g\left(x_{1}\right) e^{i \alpha x_{1}} d x_{1}
$$

Formula (21) is not applicable when $\left|x_{1}\right| \leq a$, because the short circuit in the lower complex half-plane conditions of Lemma Jordan are not met. For output expression of the displacement vector in this case, one must provide transformant from the function loads (23) in the form of the sum, which is taken in the present study as a constant on the interval $[-a, a]$ load $\mathrm{g}\left(x_{1}\right)=\mathrm{p}$ of unit cost intensity $(2 \mathrm{pa}=1)$, so it is

$$
F(\alpha)=\frac{p}{i \alpha} e^{i \alpha a}-\frac{p}{i \alpha} e^{-i \alpha a}
$$

We will substitute $F(\alpha)$ of (24) with the integral for the stripe at $x_{3}=-h$, i.e. with integral

$$
u_{3}^{(1)}\left(x_{1},-h\right)=\frac{1}{2 \pi} \int_{-\infty}^{\infty} \sum_{k=1}^{4} \frac{\tilde{D}_{k}}{D_{0}} A_{3}^{(k)} e^{-\gamma_{k} h-i \alpha x_{1}} d \alpha .
$$

Given $F(\alpha)(24)$ and the fact that

$$
\tilde{D}_{k}=(-1)^{k} D_{k} F(\alpha), \quad k=1, \ldots, 4,
$$

result the representation of $u_{3}^{(1)}\left(x_{1},-h\right)$ as a sum of two nonnative integrals. Given the above with respect to all the features of subintegral functions (25) and the types of sections, we present the final expression for $\operatorname{Im}\left(u_{3}^{(1)}\left(x_{1},-h\right)\right)$, which will be used further in the study of energy flow

$$
\operatorname{Im}\left(u_{3}^{(1)}\left(x_{1},-h\right)\right)=\operatorname{Im}\left\{i \sum_{m=1}^{N} \operatorname{res}\left[\Phi\left(\zeta_{m}^{0}\right) F\left(\zeta_{m}^{0}\right) e^{-i \zeta_{m}^{0} x_{1}}\right]+p \Phi(0)+\right.
$$

$$
\begin{gathered}
+\left.\frac{1}{2 \pi} \int_{0}^{\alpha_{1}} \Phi(\alpha) F(\alpha) e^{-i \alpha \alpha_{1}}\right|_{\left[\beta_{1}^{-}, \beta_{2}^{-}\right]} d \alpha-\left.\frac{1}{2 \pi} \int_{0}^{\alpha_{1}} \Phi(\alpha) F(\alpha) e^{-i \alpha \alpha_{1}}\right|_{\left[\beta_{1}^{+}, \beta_{2}^{+}\right]} d \alpha+(27) \\
\left.+\left.\frac{1}{2 \pi} \int_{\alpha_{1}}^{\alpha_{2}} \Phi(\alpha) F(\alpha) e^{-i \alpha \alpha_{1}}\right|_{\left[\beta_{1}^{-}, \beta_{2}^{-}\right]} d \alpha-\left.\frac{1}{2 \pi} \int_{\alpha_{1}}^{\alpha_{2}} \Phi(\alpha) F(\alpha) e^{-i \alpha \alpha_{1}}\right|_{\left[\beta_{1}^{+}, \beta_{2}^{+}\right]} d \alpha\right\} .
\end{gathered}
$$

Here were introduced the following notation:

$$
\begin{gathered}
0 \leq \alpha \leq \alpha_{1}, \quad \beta_{1}=\beta_{1}^{+}: \operatorname{Re}\left(\beta_{1}\right)>0 ; \quad \beta_{1}=\beta_{1}^{-}: \operatorname{Re}\left(\beta_{1}\right)<0, \\
\alpha_{1} \leq \alpha \leq \alpha_{2}, \beta_{1}=\beta_{1}^{-}: \quad \operatorname{Im}\left(\beta_{1}\right)<0 ; \quad\left\{\begin{array}{l}
\beta_{2}=\beta_{2}^{+}: \operatorname{Re}\left(\beta_{2}\right)>0, \\
\beta_{2}=\beta_{2}^{-}: \operatorname{Re}\left(\beta_{2}\right)<0,
\end{array}\right.
\end{gathered}
$$

where $\alpha_{1}, \alpha_{2}$ are branch points of $\beta_{1}(\alpha), \beta_{2}(\alpha)$, respectively $\left(\alpha_{1}<\alpha_{2}\right)$.

When computing now the flow of energy, «uploaded» through the loading area (22), we considered this characteristic depending on a parameter $a$ - half of the length of the segment of action of driving force In Fig. 1 one can see graphs of functions $\bar{W}(a)$ for both types of connection media. Both, here and hereafter graphics corresponding to the rigid connection are shown in solid curve by sliding dotted line. Calculations showed that $a$ in the values of $\bar{W}(a)$ is monotonically decreasing. The General predominance of flow in the case of a rigid connection there is $a$, in which this difference is maximal. When $a \rightarrow 0$, we have the following ratio of flow:

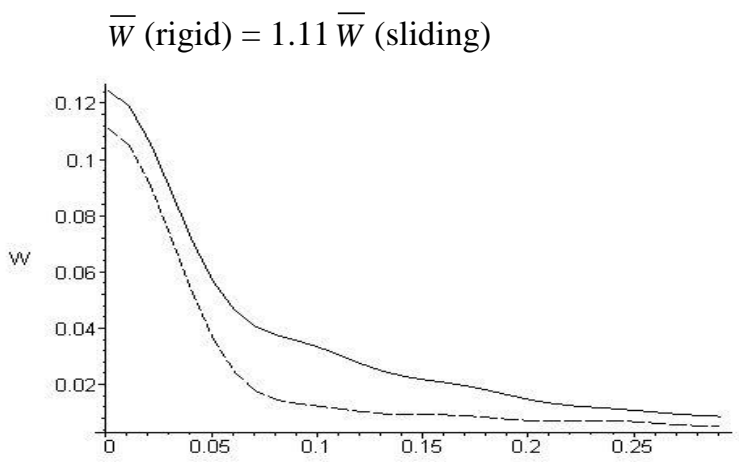

Fig. 1. Flow of energy, "uploaded" through the loading area. 
Then the flow of energy through the cross-section of the strip $\left(x_{1}=\right.$ const $)$ single width and height $h$ was considered As is known, this value is given by the formula (8):

$$
\bar{W}_{1}=\frac{i \omega}{4} \int_{-h}^{0}\left(\sigma_{11}{ }^{-(1)}-\bar{\sigma}_{11}^{-(1)} u_{1}+\sigma_{13} u_{3}^{-(1)}-\bar{\sigma}_{13} u_{3}^{-(1)}\right) d x_{3},
$$

Included in (28) stress components are expressed through the infinite integral of the band solution. After counting the stress values $\bar{W}_{1}$ has been obtained for $x_{1}>a$. The behaviour of $\bar{W}_{1}$ with growing $a$ for each connection is shown in Fig. 2. Stream values are reduced to small ones when reaching a width of $a$ of the order of the wavelength in the strip ( $a \sim \lambda=0.15$ ). With a slight predominance of $\bar{W}_{1}$ for sliding connection amplitudes take close values. When $a \rightarrow 0$, we have the following relation:

$$
\bar{W}_{1}(\text { rigid })=0.91 \bar{W}_{1}(\text { sliding }) .
$$

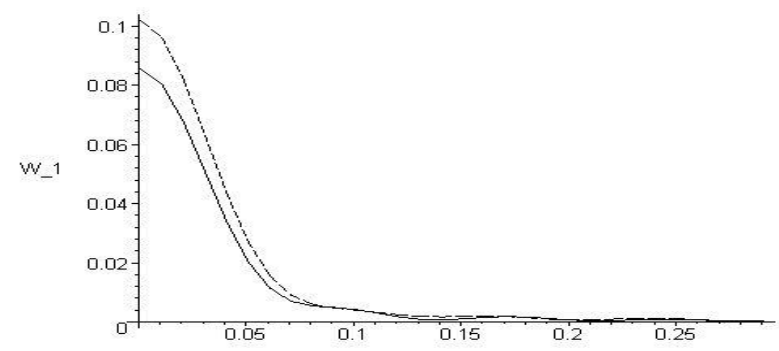

Fig. 2. Flow of energy through the cross-section of the strip.

Next, the flow of energy that propagates through the band to the half-plane is determined and the field in the half-plane is calculated based on the formula stationary phase. For this purpose the integral $\bar{u}^{-(2)}$ of (16) was introduced through the sum of Fourier integrals:

$$
\underline{u}_{2}=\underline{V}_{1}+\underline{V}_{2},
$$

where

$$
\begin{aligned}
& \underline{V}_{m}=\int_{-\infty}^{\infty} \underline{\widehat{\Phi}}(\alpha) e^{-i R q_{m}(\alpha)} d \alpha ; \\
& R=\sqrt{x_{1}^{2}+x_{3}^{2}}, \quad q_{m}(\alpha)=\alpha \sin \varphi+\beta_{m} \cos \varphi ; \\
& X_{1}=R \sin \varphi, \quad X_{3}=R \cos \varphi, \quad R>>2 \pi / q_{m}, \quad m=1,2 .
\end{aligned}
$$

Using for $\underline{V}_{m}$ of (29) the asymptotic:

$$
\underline{V}_{m} \approx \sqrt{\frac{2 \pi}{R\left|q_{m}^{\prime \prime}\left(\alpha_{c}^{m}\right)\right|}} \Phi\left(\alpha_{c}^{m}\right) e^{-i R q_{1}\left(\alpha_{c}^{m}\right)-\left.i \frac{\pi}{4} s i g n q_{m}^{\prime \prime}\right|_{\alpha=\alpha_{c}^{m}}}
$$

where $\alpha_{c}$ is nondegenerate stationary point, which is the root of the equation

$$
\frac{d q_{m}(\alpha)}{d \alpha}=0, \quad \text { while }\left.\quad \frac{d^{2} q_{1}(\alpha)}{d \alpha^{2}}\right|_{\alpha=\alpha_{c}} \neq 0 .
$$

Further, by switching to polar coordinates, the relations

$$
\hat{P}_{R}=-\frac{1}{2} \operatorname{Re}\left\{\sigma_{R R} \dot{\overline{u_{R}}}+\sigma_{R_{\varphi}} \dot{\overline{u_{\varphi}}}\right\}, \quad \bar{W}_{2}=2 \int_{0}^{\pi / 2} \widehat{P}_{R} d \varphi
$$

calculate radial component of the vector of the flow of energy $\widehat{P}_{R}$ and averaged over the period the energy stream $\bar{W}_{2}$, passing through the semicylindrical surface of the radius of $R$.

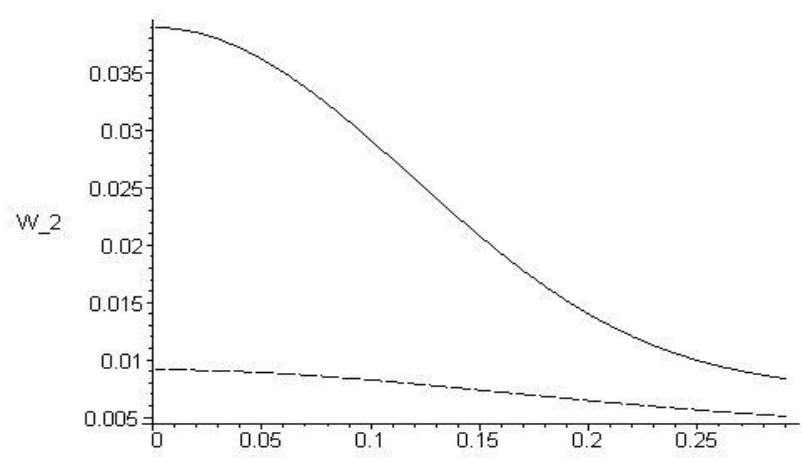

Fig. 3. Flow of energy through the band to the half-plane.

The graph of the function of the energy flow in the halfplane $\bar{W}_{2}$ is based on the same parameter as shown in Fig. 3, where there is a predominance of flow for the hard connection. When $a \rightarrow 0$ the ratio is:

$$
\bar{W}_{2}(\text { rigid })=4.26 \bar{W}_{2}(\text { sliding }) .
$$

Taking the entire portable flow $\bar{W}_{1}+\bar{W}_{2}$ for $100 \%$ with concentrated indignation, the components are separated as follows:

$$
\begin{array}{r}
\bar{W}_{1}(\text { rigid })-69 \% ; \bar{W}_{2}(\text { rigid })-31 \%=(15 \%+16 \%) \\
\bar{W}_{1}(\text { sliding })-54 \% ; \bar{W}_{2}(\text { sliding })-46 \%=(30 \%+6 \%) .
\end{array}
$$

For $\bar{W}_{2}$ the distribution is given to the parts, corresponding to quasiparallel and quasioptical waves.

\section{RESULTS AND DISCUSSION}

Thus, from the comparative analysis of properties of energy flows for two connection types it follows:

1) The total value of $\bar{W}_{1}+\bar{W}_{2}$ coincides with $\bar{W}$, which proves that the energy balance is taking place for the considered composite waveguide;

2) The available band is a barrier to the spread of the energy flow into the half-plane, so the most part of the «uploaded» power is transferred by the stripe;

3) By choosing the «moving» connection of materials the amount of power «uploaded» composite waveguide can be reduced by $10.5 \%$ when $a \rightarrow 0$ as compared with «hard» connection; 
4) It is shown that the choice of connection, can be adjusted to the distribution of the energy flow in the waveguide. In this example, the flow propagating in half-plane, while transiting from the sliding connection to the hard, increases in the band - reduces.

\section{REFERENCES}

[1] M. Babich, and A. B. Poskryakov, "About quasithin Rayleigh waves (case of anisotropic elastic body)," Zap. the scientific. SEM. POMI, vol. 332, pp. 7-18, 2006,

[2] D. P. Morgan, "A history of surface acoustic wave devices," Int. J. of High Speed Electronics and Systems, vol. 10, no. 3, pp. 553-602, 2000.

[3] V. K. Ignatovich, L. T. N. Phan, "Those wonderful elastic waves," American J. of Physics, vol. 77, № 12, pp. 1162-1172, 2009.

[4] A. H. Nikitin, P. H. Vasin, T. I. Ivankina, A. A. Kruglov, and T. Lokajicek So, "Features passing quasiparabolic elastic waves through the interface of isotropic and anisotropic media: theoretical and experimental study,” Kristallografiya, vol. 4. pp. 611-620, 2012.

[5] E. Glushkov, N. Glushkova, and A. Krivonos, "Excitation an propagation of elastic waves in layered anisotropic composites," Appl. Math. Mech., vol. 74, pp. 419-432, 2010.

[6] A. B. Belokon, and O. A. Belokon, "Harmonic oscillations in anisotropic halfplane," Izvestiya vuzov. The North Caucasus Region. Natural sciences, vol. 4. 1999.

[7] A. B. Belokon, and O. A. Belokon, "Method contour integration in the problems of harmonic oscillations of anisotropic half-plane," Izvestiva vuzov. The North Caucasus Region - Natural Science. Region. Natural Sciences, vol. 3, pp. 24-27, 2000.

[8] A. B. Belokon, and M.Y. Remizov, "Harmonic oscillations of an infinite anisotropic strip rigidly connected with an anisotropic half-plane," Dept. VINITI from 29.12.00, vol. 3324.

[9] M. Y. Remizov, "Forced oscillations of anisotropic strip rigidly associated with anisotropic half-plane," in Abstracts of the International Conference of Modern Problems of Solid Mechanics, 2000.

[10] A. Sveshnikov, "The Principle of Radiation," Dokl. The USSR Academy of Sciences, 73, vol. 5, pp. 917-920, 1950.

[11] V. Budaev, "The Roots of the characteristic equation and classification elastic anisotropic media classification," Mechanics of solids, vol. 3, 1978.

[12] V. Grinchenko, and V. Meleshko, Harmonic oscillations and waves in elastic bodies. Kiev: Naukova dumka, 1981.

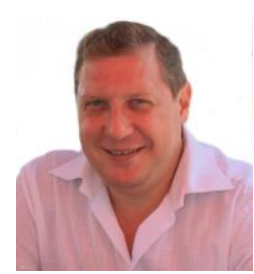

Remizov Michael received the Doctor's degree in Physics and Mathematics in 2003. He defended Doctoral Thesis "Energetic Field Analysis in Composed Anisotropic Half-Plane" in Southern Federal University, Rostov-on-Don, Russia. He is currently is an Associate Professor with the Technical Mechanics Department of Rostov-on-Don Civil Engineering University, Russia. Major areas of his research interests are: Elasticity Theory of Halfinfinite Anisotropic Composed Media; Theory of Physical Diffractions. He is the author of 30 papers published in scientific journals and presented at international and Russian scientific conferences.

E-mail: remizov72@mail.ru 